\title{
LIPSCHITZ-TYPE SPACES AND HARMONIC MAPPINGS IN THE SPACE
}

\author{
Miloš Arsenović, Vesna Manojlović and Miodrag Mateljević \\ University of Belgrade, Faculty of Mathematics \\ Studentski Trg 16, Belgrade, Serbia; arsenovic@matf.bg.ac.yu \\ University of Belgrade, Faculty of Organizational Sciences \\ Jove Ilica 154, Belgrade, Serbia; vesnak@fon.bg.ac.yu \\ University of Belgrade, Faculty of Mathematics \\ Studentski Trg 16, Belgrade, Serbia; miodrag@matf.bg.ac.yu
}

\begin{abstract}
We obtain a sharp estimate of the derivatives of harmonic quasiconformal extension $u=P[\phi]$ of a Lipschitz map $\phi: \mathbf{S}^{n-1} \rightarrow \mathbf{R}^{n}$. We also consider additional conditions which provide that $u$ is Lipschitz on the unit ball; in particular, we give characterizations of Lipschitz continuity of $u$ in the planar case and in the upper half space setting. We also answer a question posed by Martio in $[\mathrm{OM}]$ and extend this to the case of several variables.
\end{abstract}

\section{Introduction and notations}

Let $\mathbf{B}=\mathbf{B}^{n}=\left\{x \in \mathbf{R}^{n}:|x|<1\right\}$ and $\mathbf{S}=\mathbf{S}^{n-1}$ denote the unit ball and the unit sphere in $\mathbf{R}^{n}$ respectively. We write $\mathbf{U}$ and $\mathbf{T}$ instead of $\mathbf{B}^{2}$ and $\mathbf{S}^{1}$ respectively; for $r>0$, let $\mathbf{B}_{r}=\{x:|x|<r\}$ and $\mathbf{S}_{r}=\{x:|x|=r\}$.

Let $D \subset \mathbf{R}^{n}$ and $0<\alpha \leq 1$. The vector space of all functions $f: D \rightarrow \mathbf{R}^{m}$ satisfying the following condition: there is a constant $L=L_{f}$ such that $|f(x)-f(y)| \leq$ $L|x-y|^{\alpha}$ for all $x, y \in D$ is denoted by $\operatorname{Lip}(\alpha, D)$, or simply $\operatorname{Lip} \alpha$.

For $0<\alpha<1$ we say that $f \in \operatorname{Lip} \alpha$ is Hölder continuous on $D$ with exponent $\alpha$; for $\alpha=1$ we write Lip instead of $\operatorname{Lip} 1$ and we say that $f \in \operatorname{Lip}$ is Lipschitz continuous on $D$ with multiplicative (Lipschitz) constant $L=L_{f}$. Let $\Lambda_{\alpha}(D)=\Lambda_{\alpha}$ be the Banach space of all bounded Hölder continuous functions $f: D \rightarrow \mathbf{R}^{m}$ with norm

$$
\|f\|_{\alpha}=\sup _{x \in D}|f(x)|+\sup _{x, y \in D} \frac{|f(x)-f(y)|}{|x-y|^{\alpha}} .
$$

It is known, even for $n=2$, that Lipschitz continuity of $\phi: \mathbf{T} \rightarrow \mathbf{C}$, does not imply Lipschitz continuity of $u=P[\phi]$. In fact $u=P[\phi]$ is Lipschitz continuous iff the Hilbert transform of $\psi(\theta)=\frac{d}{d \theta} \phi\left(e^{i \theta}\right)$ (which is defined almost everywhere and bounded since $\phi$ is Lipschitz) is also in $L^{\infty}(\mathbf{T})$. This result is implicitly contained in [Z], see also Theorem 2.4 below. The same theorem gives additional characterizations of Lipschitz continuity of $u$ in terms of the Cauchy transform of $\psi$. A similar characterization, in terms of the Riesz transforms, is given in the setting of the upper half space $\mathbf{R}_{+}^{n+1}=\left\{(x, y): x \in \mathbf{R}^{n}, y>0\right\}$ in Theorem 3.2. In particular, $f \in C^{1, \alpha}\left(\mathbf{R}^{n}\right)$

doi:10.5186/aasfm.2010.3524

2000 Mathematics Subject Classification: Primary 30C80, 30C62; Secondary 30C55, 30H05.

Key words: Lipschitz-type spaces, harmonic mappings, quasiregular mappings.

Research partially supported by MNTRS, Serbia, Grant No. 144020. 
implies $u=P[f] \in C^{1, \alpha}\left(\mathbf{R}_{+}^{n+1}\right)$. Here, for any $n \geq 2$,

$$
P[\phi](x)=\int_{S^{n-1}} P(x, \xi) \phi(\xi) d \sigma(\xi), \quad x \in \mathbf{B}^{n},
$$

where $P(x, \xi)=\frac{1-|x|^{2}}{|x-\xi|^{n}}$ is the Poisson kernel for the unit ball $\mathbf{B}^{n}, d \sigma$ is the normalized surface measure on the unit sphere $\mathbf{S}^{n-1}$ and $\phi: \mathbf{S}^{n-1} \rightarrow \mathbf{R}^{n}$ is a continuous mapping. The corresponding formula for the upper half space is

$$
P[\phi](x, y)=\int_{\mathbf{R}^{n}} P(x-t, y) \phi(t) d t
$$

where

$$
P(x, y)=c_{n} \frac{y}{\left(|x|^{2}+y^{2}\right)^{n+1 / 2}}, \quad c_{n}=\Gamma\left(\frac{n+1}{2}\right) \pi^{-(n+1) / 2},
$$

is the Poisson kernel for the upper half space. The Riesz transforms $R_{j}, 1 \leq j \leq n$, in $\mathbf{R}^{n}$ are defined by principal value integrals

$$
R_{j} f(x)=c_{n} \int_{\mathbf{R}^{n}} \frac{x_{j}-y_{j}}{|x-y|^{n+1}} f(y) d y,
$$

they are bounded on $L^{p}\left(\mathbf{R}^{n}\right)(1<p<\infty)$ and $\Lambda_{\alpha}\left(\mathbf{R}^{n}\right)(0<\alpha<1)$ spaces. It is important to note that these operators are not bounded on $L^{1}\left(\mathbf{R}^{n}\right), L^{\infty}\left(\mathbf{R}^{n}\right)$ and $\Lambda_{1}\left(\mathbf{R}^{n}\right)$. We refer to [St] for a detailed discussion of these results in the context of singular integral operators.

Similar results hold in the $\mathbf{S}^{n-1}$ setting. Indeed, Hölder continuity of $\phi: \mathbf{S}^{n-1} \rightarrow$ $\mathbf{R}^{n}$ with exponent $\alpha, 0<\alpha<1$, implies Hölder continuity of its harmonic extension $u=P[\phi]$, see $[\mathrm{Dy}],[\mathrm{NO}]$. In the case $n=2$ it is a classical result, following from Privalov's theorem (see [Z]).

In Section 3, using the maximum principle for harmonic functions, we prove:

Claim 1. If $\phi: \mathbf{S}^{n-1} \rightarrow \mathbf{R}^{n}$ is Lipschitz continuous with Lipschitz constant $L_{\phi}=L$, then $u=P[\phi]$ is Lipschitz continuous with constant $L_{u}=L / r$ on the spheres $\mathbf{S}_{r}, 0<r<1$.

In the case $n=2$, using Schwarz lemma for harmonic functions, we prove an estimate $\left|\partial_{\theta} h(z)\right| \leq \frac{4}{\pi} L|z|$ (see Theorem 2.1).

Harmonic quasiconformal mappings were first studied by Martio in [OM]. Now it is a very active area of investigation (see [K3]). The following theorem has recently been proved in $[\mathrm{AKM}]$ :

Theorem 1.1. Assume $\phi: \mathbf{S}^{n-1} \rightarrow \mathbf{R}^{n}$ satisfies a Lipschitz condition

$$
|\phi(\xi)-\phi(\eta)| \leq L|\xi-\eta|, \quad \xi, \eta \in \mathbf{S}^{n-1},
$$

and assume its harmonic extension $u=P[\phi]: \mathbf{B}^{n} \rightarrow \mathbf{R}^{n}$ is $K$-quasiregular. Then

$$
|u(x)-u(y)| \leq C^{\prime}|x-y|, \quad x, y \in \mathbf{B}^{n},
$$

where $C^{\prime}$ depends on $L, K$ and $n$ only.

Kalaj obtained a related result, but under additional assumption of $C^{1, \alpha}$ regularity of $\phi$, see [K1]. In the case $n=2$ this assumption (without hypothesis that $u$ is $K$-quasiregular) implies that partial derivatives of $u$ are Hölder continuous and, in particular, that $u$ is Lipschitz on $\mathbf{U}$ (see Theorem 2.3). 
The proof of Theorem 1.1 was based on estimates of the gradient of the Poisson integral kernel and did not yield sharp bounds on $C^{\prime}$. We give another proof of this result, based on application of the maximum principle to a subharmonic function $A(x, a)=\sum_{\nu=1}^{n}\left|d h_{\nu}(x) a\right|^{2}$ and on Claim 1, where $a$ is a unit vector. Using this approach we obtain $C^{\prime}=K L$ (a dimension-free estimate).

\section{The planar case}

In the planar case we use the notation $z=r e^{i \theta}$. If $h$ is a function of variable $z$, we consider also $h$ as a function of variables $(r, \theta)$ (polar coordinates). Also, for $f: \mathbf{T} \rightarrow \mathbf{C}$, we define $\hat{f}$ on $[0,2 \pi]$ by $\hat{f}(t)=f\left(e^{i t}\right)$.

The following fact will be used below: if $h$ is harmonic in $\mathbf{U}$, then $r \partial_{r} h$ is the harmonic conjugate of $\partial_{\theta} h$.

We refer the reader to $[\mathrm{Du}]$ for an excellent exposition on harmonic mappings in the plane, see also $[\mathrm{BH}]$.

It is known that Privalov theorem for harmonic functions with $C^{\alpha}$ boundary values, $0<\alpha<1$, fails for $\alpha=1$. The next theorem deals with the case $\alpha=1$ and explains that this failure is due to the loss of control of the radial derivative, see also $[\mathrm{K}]$.

Theorem 2.1. Suppose that $h$ is a harmonic mapping from $\mathbf{U}$ continuous on $\overline{\mathbf{U}}$. Then the following conditions are equivalent:

a)

$$
\left|h\left(e^{i \theta_{1}}\right)-h\left(e^{i \theta_{2}}\right)\right| \leq m\left|\theta_{1}-\theta_{2}\right| .
$$

b)

$$
\left|h^{\prime}(z) T\right| \leq M
$$

for every $z \in \mathbf{U}$ and unit vector $T=i e^{i \theta}$ which is tangent to the circle $\mathbf{S}_{r}$ at $z=r e^{i \theta}$.

c) $\partial_{\theta} h$ is bounded on $\mathbf{U}$.

a) implies b) with constant $M=\frac{4}{\pi} m$. b) implies a) with constant $m=M$.

Proof. Suppose that a) holds. Then $\mid \frac{d}{d \theta} h\left(e^{i \theta} \mid \leq m\right.$ a.e. Since $\hat{h}$ is absolutely continuous on $[0,2 \pi]$ and $\partial_{\theta} P_{r}(\theta-t)=-\partial_{t} P_{r}(\theta-t)$, using integration by parts, we have

$$
\partial_{\theta} h(z)=-\frac{1}{2 \pi} \int_{0}^{2 \pi} \partial_{t} P_{r}(\theta-t) h\left(e^{i t}\right) d t=\frac{1}{2 \pi} \int_{0}^{2 \pi} P_{r}(\theta-t) d \hat{h}=P\left[\hat{h}^{\prime}\right] .
$$

Therefore, by Proposition 3.2, $\left|\partial_{\theta} h(z)\right| \leq m, z \in \mathbf{U}$. Since $\partial_{\theta} h(0)=0$, by the harmonic version of Schwarz lemma, we find $\left|h_{\theta}^{\prime}(z)\right| \leq \frac{4}{\pi} m|z|$.

Now the estimate (2), with $M=\frac{4}{\pi} m$, follows easily. The remaining straightforward implications are left to the reader.

An easy corollary of the above theorem is the following result:

Proposition 2.1. Suppose that $h$ is a harmonic quasiregular map in U. Then the following conditions are equivalent:

(1) $h$ is Lipschitz continuous on $\mathbf{U}$.

(2) $h$ has continuous extension to $\overline{\mathbf{U}}$ which belongs to Lip on $\mathbf{T}$.

(3) $\operatorname{grad} h$ is bounded on $\mathbf{U}$, i.e. $|\operatorname{grad} h(z)| \leq A, z \in \mathbf{U}$. 
For a function $f$ defined on $\mathbf{U}$, we define

$$
f_{*}(\theta)=f^{*}\left(e^{i \theta}\right)=\lim _{r \rightarrow 1} f\left(r e^{i \theta}\right)
$$

whenever the limit exists.

If $\psi \in L^{1}[0,2 \pi]$, the Cauchy transform $C[\psi]$ of $\psi$ is defined by

$$
C[\psi](z)=\frac{1}{2 \pi} \int_{0}^{2 \pi} \frac{\psi(t) e^{i t}}{e^{i t}-z} d t
$$

and the Hilbert transform of $\psi$ is defined by

$$
H \psi(\theta)=\frac{1}{\pi} \int_{-\pi}^{\pi} \frac{\psi(\theta-t) d t}{\tan (t / 2)},
$$

where the integral is interpreted in the principal value sense.

In $[\mathrm{OM}]$ the following situation was considered: $u$ is a harmonic function in the unit disc which assumes continuous boundary values $f$ on $\mathbf{T}, u_{r}$ and $u_{\theta}$ are derivatives of $u$ with respect to $r$ and $\theta$. A question posed in that paper is: find necessary and sufficient conditions ensuring that the $\operatorname{limits}_{\lim _{z \rightarrow \zeta}} u_{r}(z)=\phi(\zeta)$ and $\lim _{z \rightarrow \zeta} u_{\theta}(z)=\psi(\zeta)$ exist at each boundary point $\zeta \in \mathbf{T}$. The following proposition answers that question, a related problem in higher dimensions is discussed in the next section.

Proposition 2.2. In the above situation, both limits $\lim _{z \rightarrow \zeta} u_{r}(z)=\phi(\zeta)$ and $\lim _{z \rightarrow \zeta} u_{\theta}(z)=\psi(\zeta)$ exist at each boundary point $\zeta \in \mathbf{T}$ if and only if $\hat{f}(t)=f\left(e^{i t}\right)$ is continuously differentiable and $H\left[\hat{f}^{\prime}\right]$ is continuous.

Proof. Assume that the two limits exist at each boundary point, then they define continuous functions $\psi(t)=\lim _{z \rightarrow e^{i t}} u_{\theta}(z)$ and $\phi(t)=\lim _{z \rightarrow e^{i t}} u_{r}(z)$. Therefore $u_{\theta}\left(r e^{i t}\right)$ converges uniformly over $t$ as $r \rightarrow 1$, which shows that $\hat{f}$ is a $C^{1}$ function and $u_{\theta}\left(r e^{i t}\right) \rightrightarrows \hat{f}^{\prime}(t)$ as $r \rightarrow 1$. Similarly, $u_{r}\left(r e^{i t}\right)$ converges uniformly over $t$ as $r \rightarrow 1$ to a continuous function $g(t)$, hence $r u_{r}\left(r e^{i t}\right) \rightrightarrows g(t)$ as $r \rightarrow 1$. However, $r u_{r}$ is the harmonic conjugate of $u_{\theta}$ and therefore the corresponding boundary functions are related by the Hilbert transform: $H\left[\hat{f}^{\prime}\right]=g$.

Conversely, if $f$ is $C^{1}$ and $H\left[\hat{f}^{\prime}\right]=g$ is continuous, then the harmonic extension of $\hat{f}^{\prime}$ is equal to $u_{\theta}$ and the harmonic extension of $g$ is the harmonic conjugate of $u_{\theta}$, that is, $r u_{r}$. Hence both $u_{\theta}$ and $r u_{r}$, and therefore $u_{r}$ as well, have continuous extension to the boundary.

In fact, in $[\mathrm{OM}]$, boundary functions $f$ of the form $\hat{f}(t)=f\left(e^{i t}\right)=e^{i \chi(t)}$ were considered, where $\chi$ is a continuous increasing function on $\mathbf{R}$ such that $\chi(t+2 \pi)=$ $\chi(t)+2 \pi$ and the characterization problem was posed in terms of the function $\chi$.

Theorem 2.2. In the above situation, the limits $\lim _{z \rightarrow \zeta} u_{r}(z)=\phi(\zeta)$ and $\lim _{z \rightarrow \zeta} u_{\theta}(z)=\psi(\zeta)$ exist at each boundary point $\zeta \in \mathbf{T}$ if and only if $\chi(t)$ is continuously differentiable and $H\left[\chi^{\prime}\right]$ is continuous.

Proof. Since $\hat{f}$ is $C^{1}$ if and only if $\chi$ is $C^{1}$, in view of the above proposition it suffices to prove the following statement: if $\chi$ is $C^{1}$, then $H\left[\hat{f}^{\prime}\right]$ is continuous if and only if $H\left[\chi^{\prime}\right]$ is continuous. Indeed, we have

$$
H\left(\hat{f}^{\prime}\right)(\theta)=\frac{1}{\pi} \int_{-\pi}^{\pi} \frac{\hat{f}^{\prime}(\theta-t) d t}{\tan (t / 2)}=-\frac{1}{\pi} \int_{+0}^{\pi} \frac{\hat{f}(\theta+t)+\hat{f}(\theta-t)-2 \hat{f}(\theta)}{2 \sin ^{2}(t / 2)} d t
$$


almost everywhere and therefore

$$
e^{-i \chi(\theta)} H\left(\hat{f}^{\prime}\right)(\theta)=-\frac{1}{\pi} \int_{0}^{\pi} \frac{e^{i(\chi(\theta+t)-\chi(\theta))}+e^{i(\chi(\theta-t)-\chi(\theta))}-2}{2 \sin ^{2}(t / 2)} d t .
$$

Define $S(\theta, t)=\sum_{k=2}^{\infty} a_{k}(\theta, t)$, where

$$
a_{k}(\theta, t)=i^{k} \frac{(\chi(\theta+t)-\chi(\theta))^{k}+(\chi(\theta-t)-\chi(\theta))^{k}}{k ! \sin ^{2}(t / 2)} .
$$

Each of the functions $a_{k}(\theta, t), k \geq 2$, is continuous and there is a constant $C$ independent of $k$ such that $\left|a_{k}(\theta, t)\right| \leq C^{k-2} / k$ ! for $k \geq 2$. Therefore, $S$ is a continuous function and $\int_{0}^{\pi} S(\theta, t) d t$ is a continuous function of $\theta$.

Hence, using $e^{i u}=1+i u+E(u)$, where $E(u)=\sum_{k=2}^{\infty}(i u)^{k} / k$ !, we find

$$
e^{-i \chi(\theta)} H\left(\hat{f}^{\prime}\right)(\theta)=i H\left(\chi^{\prime}\right)(\theta)+R(\theta),
$$

where $R$ is a continuous function. Hence, $H\left(\hat{f}^{\prime}\right)$ is continuous if and only if $H\left(\chi^{\prime}\right)$ is continuous.

Suppose that $\phi$ is Lipschitz on $\mathbf{T}$ and $h=P[\phi]$. Then $\partial_{\theta} h$ is bounded on $\mathbf{U}$ and $\partial_{r} h \in H^{p}, 0<p<\infty$; hence $\left(\partial_{r} h\right)^{*}$ exists a.e. on $\mathbf{T}$. Using a routine argument one can show that $\partial_{r} h\left(e^{i t}\right)$ exists a.e. and $\left(\partial_{r} h\right)^{*}\left(e^{i t}\right)=\partial_{r} h\left(e^{i t}\right)$ a.e. on $\mathbf{T}$, where

$$
\partial_{r} h\left(e^{i t}\right)=\lim _{r \rightarrow 1-0} \frac{h^{*}\left(e^{i t}\right)-h\left(r e^{i t}\right)}{1-r} .
$$

We say that $\phi \in C^{1, \alpha}(\mathbf{T}), 0<\alpha<1$, if $\hat{\phi}^{\prime}$ belongs to Lip $\alpha$ on $[0,2 \pi]$.

In the next two theorems we use the following representation of a complex valued harmonic function $h$ on $\mathbf{U}: h=f+\bar{g}$, where $f$ and $g$ are analytic. Note that $f$ and $g$ are unique, up to an additive constant.

Theorem 2.3. If $\phi \in C^{1, \alpha}(\mathbf{T}), 0<\alpha<1$, and $h=P[\phi]$, then

a) $\partial_{\theta} h$ and $\partial_{r} h$ belong to $\operatorname{Lip} \alpha$ on $U$, and

b) $\left|f^{\prime \prime}(z)\right|+\left|g^{\prime \prime}(z)\right|=O(1-r)^{\alpha-1}, z \in \mathbf{U}$, where $r=|z|$.

In particular, $h$ is Lipschitz on $\mathbf{U}$.

Proof. Since $\hat{\phi}$ is absolutely continuous on $[0,2 \pi]$, we find

$$
\partial_{\theta} h(z)=\frac{1}{2 \pi} \int_{0}^{2 \pi} P_{r}(\theta-t) d \hat{\phi}(t)=\frac{1}{2 \pi} \int_{0}^{2 \pi} P_{r}(\theta-t) \hat{\phi}^{\prime}(t) d t .
$$

Hence, by Privalov's theorem, $\partial_{\theta} h$ belongs to Lip $\alpha$ on U. Since Hölder continuity of a harmonic function implies Hölder continuity of its harmonic conjugate, we conclude that $\partial_{r} h$ belongs to $\operatorname{Lip} \alpha$ on $\mathbf{U}$; therefore $f^{\prime}$ and $g^{\prime}$ belong to $\operatorname{Lip} \alpha$ on $\mathbf{U}$ and we get b).

In particular, $\operatorname{grad} h$ is bounded on $\mathbf{U}$ and therefore $h$ is Lipschitz on $\mathbf{U}$.

Theorem 2.4. Suppose that $\phi$ is Lipschitz on $\mathbf{T}$ and $h=P[\phi]$. Then the following conditions are equivalent:

(1) $h$ is Lipschitz on $\mathbf{U}$.

(2) The Cauchy transform $C\left[\hat{\phi}^{\prime}\right]$ is bounded.

(3) The Cauchy transform $C\left[\hat{\phi}^{\prime}\right]$ is bounded.

(4) $\left(\partial_{r} h\right)^{*}$ is bounded on $\mathbf{T}$. 
(5) $\partial_{r} h\left(e^{i t}\right)$ is bounded on $\mathbf{T}$.

(6) $\left|f^{\prime}(z)\right|+\left|g^{\prime}(z)\right|$ is bounded on $\mathbf{U}$.

Proof. Since $\phi$ is Lipschitz on $\mathbf{T}, \phi$ is absolutely continuous, and then $C\left[\phi^{\prime}\right](z)=$ $i z f^{\prime}(z)$ and $C\left[\overline{\hat{\phi}^{\prime}}\right](z)=i z g^{\prime}(z)$, where $C$ is the Cauchy transform and $i z f^{\prime}(z)$ is the analytic part of $\partial_{\theta} h$.

Since, by Theorem 2.1, $\partial_{\theta} h(z)=i\left(z f^{\prime}(z)-\overline{z g^{\prime}(z)}\right)$ is bounded on $\mathbf{U}$, we see at once that (2), (3) and (6) are equivalent.

If (3) holds, then $\partial_{r} h$ is bounded on $\mathbf{U}$. The rest of the proof is routine.

\section{Higher dimensions}

Now we turn to the general case.

Let $f$ be a vector-valued function defined in a neighborhood of a point $z \in \mathbf{R}^{n}$, differentiable at $z$. By $f^{\prime}(z)$ we denote the linear operator $d f(z)$ between the tangent spaces at $z$ and $f(z)$.

For linearly independent $x, y \in \mathbf{R}^{n}$, we denote by $L(x, y)$ the plane defined by 0 , $x$ and $y$. We can choose an orthonormal base $e_{1}, \ldots, e_{n}$ such that $L(x, y)=L\left(e_{1}, e_{2}\right)$. For $z=\left(z_{1}, \ldots, z_{n}\right)=\sum_{k=1}^{n} z_{k} e_{k}$ define $P z=z_{1} e_{1}+z_{2} e_{2}$ and $Q z=z_{3} e_{3}+\cdots+z_{n} e_{n}$. If $\alpha$ is the oriented angle between $x$ and $y$, we define the rotation $R=R_{x, y}$ by $R z=\left(e^{i \alpha} P z, Q z\right)$. Hence $R$ is in the orthogonal group $O(n)$, acts as the identity map on the orthocomplement of $L(x, y)$ and, in the case $|x|=|y|$, maps $x$ to $y$.

For $x \in \mathbf{R}^{n}, x \neq 0$, we set $x^{*}=\frac{x}{|x|} \in \mathbf{S}$. Note that $|R z-z|=\left|e^{i \alpha} P z-P z\right|=$ $|P z|\left|1-e^{i \alpha}\right| \leq\left|x^{*}-y^{*}\right|$ for $z \in \overline{\mathbf{B}}$; and if $|x|=|y|$, then $R x=y$ and $R x^{*}=y^{*}$. Thus

$$
\max \{|R z-z|: z \in \mathbf{S}\} \leq\left|x^{*}-R x^{*}\right|=\left|x^{*}-y^{*}\right| .
$$

Since the Laplacian commutes with orthogonal transformations [ABR, pp. 3-4], we have:

Proposition 3.1. If $h: \mathbf{B}^{n} \rightarrow \mathbf{R}^{n}$ is harmonic on $\mathbf{B}^{n}$, then $h \circ R$ is harmonic.

Proposition 3.2. Suppose that $h$ is a harmonic mapping from $\mathbf{B}^{n}$ continuous on $\overline{\mathbf{B}}^{n}$ and $M=\max \{|h(t)|: t \in \mathbf{S}\}$. Then $|h(t)| \leq M$ for $|t| \leq 1$.

A proof can be based on the Poisson representation, or, alternatively, reduced (using scalar product) to the classical real valued case.

We will also use a version of Harnack's inequality (see [MV]): Let $B=B(a ; r) \subset$ $\mathbf{R}^{n}$ be a ball. Suppose $h: B \rightarrow \mathbf{R}^{n}$ is a vector valued harmonic mapping on $B$ and $M_{a}=\sup \{|h(y)-h(a)|: y \in B\}$. Then

$$
r\left|h^{\prime}(a)\right| \leq n M_{a} .
$$

Conjecture 1. Let $D$ be a domain in $\mathbf{R}^{n}$ with $C^{1}$ (or $C^{\infty}$ ) boundary, let $y_{0} \in D$ and let $g(x)=g\left(x, y_{0}\right)$ be the Green's function for $D$. Set $S_{c}=\{x \in D: g(x)=c\}$. Suppose that $h: D \rightarrow \mathbf{R}^{m}$ is a harmonic mapping which is continuous on $\bar{D}$. The following conditions are equivalent:

a) $h$ is Lipschitz on $\partial D$.

b)

$$
\left|h^{\prime}(x) T\right| \leq M
$$

for every $x \in D$ and unit vector $T$ which is tangent to $S_{c}, c>0$, at $x$.

We prove this conjecture for the unit ball, taking $y_{0}=0$. 
Theorem 3.1. Suppose that $h: \overline{\mathbf{B}}^{n} \rightarrow \mathbf{R}^{n}$ is harmonic on $\mathbf{B}^{n}$ and continuous on $\overline{\mathbf{B}}^{n}$. Then the following conditions are equivalent:

a)

b)

$$
|h(x)-h(y)| \leq L|x-y|, x, y \in \mathbf{S}
$$

$$
\left|h^{\prime}(x) T\right| \leq M
$$

for every $x \in \mathbf{B}^{n}$ and unit vector $T$ which is tangent on $\mathbf{S}_{r}$, where $r=|x|$.

If we suppose, in addition, that $h$ is K-quasiregular mapping, then

c)

$$
\left|h^{\prime}(x)\right| \leq K L
$$

for every $x \in \mathbf{B}^{n}$.

Proof. It is clear that b) implies a), with constant $L=M$.

Suppose that a) holds. Let $x_{0} \in \mathbf{S}$ be fixed. Then $\left|h(x)-h\left(x_{0}\right)\right| \leq C_{1}=2 L$ on $\mathbf{S}$ and by Poisson representation $\left|h(x)-h\left(x_{0}\right)\right| \leq C_{1}=2 L$ on B. Using translation, we can suppose that $h\left(x_{0}\right)=0$. Hence $|h(x)-h(a)| \leq C_{2}=4 L$ for $x, a \in \mathbf{B}$.

If $|x| \leq 1 / 2$, then an application of $(3.2)$ on the ball $\mathbf{B}(x ; 1 / 2)$ gives $\left|h^{\prime}(x)\right| \leq$ $2 n C_{2}$. Hence there is a constant $C_{3}=2 n C_{2}=8 n L$ such that

$$
|h(x)-h(y)| \leq C_{3}|x-y|
$$

for every $x, y \in \mathbf{B}_{1 / 2}$.

Let us prove that

$$
|h(x)-h(y)| \leq L\left|x^{*}-y^{*}\right|=L_{r}|x-y|, \quad|x|=|y|=r,
$$

where $L_{r}=L / r$.

Let $R=R_{x, y}$ be the rotation described above which maps $x$ to $y$. Note that $\max \{|R z-z|: z \in \mathbf{S}\} \leq\left|x^{*}-y^{*}\right|$. By Proposition 3.1, the function $h(z)-h(R z)$ is harmonic in $z$. By hypothesis a), $|h(z)-h(R z)| \leq L|R z-z|, z \in \mathbf{S}$. Hence, by (3.1), $\max \{|h(z)-h(R z)|: z \in \mathbf{S}\} \leq L\left|x^{*}-R x^{*}\right|$. Now applying Proposition 3.2 (the maximum principle), we conclude that $|h(x)-h(R x)| \leq L_{r}|x-R x|$. Thus $|h(x)-h(y)| \leq L_{r}|x-y|$ whenever $|x|=|y|=r<1$. Clearly this proves the following estimate:

$$
\left|h^{\prime}(x) T\right| \leq L_{r}
$$

for every $x \in \mathbf{B}^{n}$ and unit vector $T$ which is tangent to $\mathbf{S}_{r}$, where $r=|x|$. In particular, for $r \geq 1 / 2,\left|h^{\prime}(x) T\right| \leq 2 L$. By (3.3), we can choose $M=8 n L$.

Now we prove c). Let $a \in \mathbf{S}^{n-1}$ be a fixed unit vector. Then the function $A(x, a)=|d h(x) a|^{2}=\sum_{\nu=1}^{n}\left|d h_{\nu}(x) a\right|^{2}$ is subharmonic in $x \in \mathbf{B}$. Using estimate (3.4) and quasiregularity of $h$ we obtain $|d h(x) a| \leq K L / \rho$ on $\mathbf{S}_{\rho}, 0<\rho<1$. Now the maximum principle for subharmonic functions gives, as $\rho \rightarrow 1,|d h(x) a| \leq K L$ on $\mathbf{B}$, and since $a$ is an arbitrary unit vector we conclude $\left|h^{\prime}(x)\right| \leq K L$.

One consequence of the tangential estimate (3.4) is:

Proposition 3.3. Suppose that $\phi: \mathbf{S}^{n-1} \rightarrow \mathbf{R}^{n}$ is Lipschitz. Let $u=P[\phi]$. Then the following conditions are equivalent:

(1) $u=P[\phi]: \mathbf{B}^{n} \rightarrow \mathbf{R}^{n}$ is Lipschitz on $\mathbf{B}^{n}$.

(2) The radial derivative of $u$ is bounded on $\mathbf{B}^{n}$.

(3) $\operatorname{grad} u$ is bounded on $\mathbf{B}^{n}$. 
Next we consider the upper half space $\mathbf{R}_{+}^{n+1}$ setting. First, we note that a harmonic map $u: \mathbf{R}_{+}^{n+1} \rightarrow \mathbf{R}^{n+1}$ is bounded and extends continuously to the boundary if and only if $u=P[f]$ for some bounded continuous map $f: \mathbf{R}^{n} \rightarrow \mathbf{R}^{n+1}$. In this case $f$ is Lipschitz continuous if and only if the partial derivatives $\partial_{j} u, 1 \leq j \leq n$, are bounded on $\mathbf{R}_{+}^{n+1}$. This is, of course, a necessary condition for Lipschitz continuity of $u$. To get a necessary and sufficient condition, one has to ensure that $\partial u / \partial y$ is bounded as well. Let $\mathscr{F} f(\xi)$ be the Fourier transform of $f$ in the sense of distributions, i.e., $\mathscr{F} f$ is in the space $\mathscr{S}^{\prime}$ of tempered distributions. Then $i \xi_{j} \mathscr{F} f(\xi)$ is the Fourier transform of $\partial_{j} f$ for $1 \leq j \leq n$. Also, the Fourier transform of $u(x, y)$ for a fixed $y>0$ is $e^{-y|\xi|} \mathscr{F} f(\xi)$ and therefore the Fourier transform of $\partial_{y} u(x, y)$ is $-|\xi| e^{-y|\xi|} \mathscr{F} f(\xi)$. Hence, taking the limit $y \rightarrow 0$ in $\mathscr{S}^{\prime}$, we see that the boundary values $g$ of $\partial u / \partial y$ satisfy $\mathscr{F} g(\xi)=-|\xi| \mathscr{F} f(\xi)$. Therefore, $\mathscr{F}\left(\partial_{j} f\right)(\xi)=-i \xi_{i} /|\xi| \mathscr{F} g(\xi)$ which means that $\partial_{j} f=R_{j} g$, where $R_{j}$ denotes the Riesz transform. We can summarize the above discussion in the following theorem.

Theorem 3.2. A harmonic map $u: \mathbf{R}_{+}^{n+1} \rightarrow \mathbf{R}^{n+1}$ is bounded and Lipschitz continuous if and only if $u=P[f]$, where $f$ is bounded and Lipschitz continuous on the boundary $\mathbf{R}^{n}, \phi_{j}=\partial f / \partial x_{j}$ are in $L^{\infty}\left(\mathbf{R}^{n}\right)$ for all $1 \leq j \leq n$ and for some (equivalently all) $j$ the function $\phi_{j}$ is the $R_{j}$ transform of a function in $L^{\infty}\left(\mathbf{R}^{n}\right)$.

Note that the theorem remains valid, with essentially the same proof, if one replaces "bounded and Lipschitz continuous" with "bounded with continuous and bounded partial derivatives" and $L^{\infty}\left(\mathbf{R}^{n}\right)$ with $B C\left(\mathbf{R}^{n}\right)$ (the space of continuous and bounded functions on $\mathbf{R}^{n}$ ). This extends Proposition 2.2 to the multidimensional case.

It is easy to derive sufficient conditions from the above result: since the Riesz transforms $R_{j}$ preserve $\Lambda_{\alpha}$ spaces, $0<\alpha<1$, any bounded function with partial derivatives in $\Lambda_{\alpha}$ extends to a Lipschitz continuous harmonic function in the upper half space, in fact that extension is in $C^{1, \alpha}\left(\mathbf{R}_{+}^{n+1}\right)$.

\section{References}

[Ahl] Ahlfors, L.: Möbius transformation in several dimensions. - School of Mathematics, University of Minnesota, 1981.

[AKM] Arsenović, M., V. Kojić, and M. Mateljević On Lipschitz continuity of harmonic quasiregural mappings on the unit ball in $\mathbf{R}^{n}$. - Ann. Acad. Sci. Fenn. Math. 33, 2008, $315-318$.

[ABR] Axler, S., P. Bourdon, and W. Ramey: Harmonic function theory. - Springer-Verlag, New York, 1992.

[BH] Bshouty, D., and W. Hengartner: Univalent harmonic mappings in the plane. - In: Handbook of Complex Analysis: Geometric Function Theory, Volume 2, edited by R. Kühnau, 2005, 479-506.

[Du] Duren, P.: Harmonic mappings in the plane. - Cambridge Univ. Press, 2004.

[Dy] Dyakonov, K. M.: Holomorphic functions and quasiconformal mappings with smooth moduli. - Adv. Math. 187:1, 2004, 146-172.

[IM] Iwaniec, T., and G. Martin: Geometric function theory and nonlinear analysis. - Syracuse and Auckland, 2000.

[K] KALAJ, D.: Harmonijske funkcije i kvazikonformna preslikavanja (Harmonic functions and quasiconformal mappings). - Master thesis, 1998 (in Serbian). 
[K1] KalAJ, D.: On harmonic quasiconformal self-mappings of the unit ball. - Ann. Acad. Sci. Fenn. Math. 33, 2008, 261-271.

[K2] KALAJ, D.: Quasiconformal harmonic mapping between Jordan domains. - Math. Z. 260:2, $2008,237-252$.

[K3] KalaJ, D.: Lipschitz spaces and harmonic mappings. - Ann. Acad. Sci. Fenn. Math. 34:2, $2009,475-485$.

[KM] Kalaj, D., and M. Mateljević: Inner estimate and quasiconformal harmonic maps between smooth domains. - J. Anal. Math. 100, 2006, 117-132.

[MV] Manojlović, V.: Bi-Lipschicity of quasiconformal harmonic mappings in the plane. Filomat 23:1, 2009, 85-89.

[OM] Martio, O.: On harmonic quasiconformal mappings. - Ann. Acad. Sci. Fenn. Ser. A I Math. 425, 1968, 3-10.

[M] Mateljević, M.: Distortion of harmonic functions and harmonic quasiconformal quasiisometry. - Rev. Roumaine Math. Pures Appl. 51:5-6, 2006, 711-722.

[M1] MatelJević, M.: Lipschitz-type spaces and quasiregular harmonic mappings in the space and applications. - Manuscript, 2007.

[M2] Mateljević, M.: On quasiconformal harmonic mappings. - Manuscript, 2006.

[MV] MatelJević, and M. Vuorinen: On harmonic quasiconformal quasi-isometries. - arXiv: $0709.4546 \mathrm{v} 1$.

[NO] Nolder, C. A., and D. M. Oberlin: Modulus of continuity and a Hardy-Littlewood theorem. - Lecture Notes in Math. 1351, 1988, 265-272.

[Ri] Rickman, S.: Quasiregular mappings. - Springer-Verlag, Berlin Heidelberg, 1993.

[St] Stein, E.: Singular integrals and differentiability properties of functions. - Princeton Univ. Press, 1970.

[Z] Zygmund, A.: Trigonometrical series. - Chelsea Publishing Co., 2nd ed., New York, 1952.

Received 8 May 2009 